\title{
GROUP-SPECIFIC COMPONENT (Gc) POLYMORPHISM IN JAPANESE: AN ANALYSIS BY ISOELECTRIC FOCUSING ON POLYACRYLAMIDE GELS ${ }^{1}$
}

\author{
Goichi Ishimoto, * Mieko Kuwata, * and Hachiro NakajImA** \\ * Department of Legal Medicine, Mie University School of Medicine, Tsu \\ **Department of Forensic Medicine, Tokyo Medical and Dental \\ University, Tokyo
}

\begin{abstract}
Summary Using the immunofixation isoelectric focusing method, Gc subtypes were examined in the samples with the $\mathrm{Gc} J$ phenotypes previously determined by immunoelectrophoresis, and it was found that the $G c^{\text {s }}$ s are subdivided into 4 different alleles. A population study in sera from 1,041 individuals confirmed the presence of the 4 variant alleles with the frequencies between 0.020 for the highest allele and 0.001 for the lowest one. The distribution of Gc subtypes in a Japanese population and a pedigree study from 18 families with the propositus of the variant are presented along with a description of the 2 other variants observed in the course of this study.
\end{abstract}

\section{INTRODUCTION}

Recently, Constans and Viau (1977) developed a new technique to detect a genetic variation of the group-specific component(Gc) of human serum and demonstrated the presence of heterogeneity in the Gc 1 protein. Using isoelectric focusing on polyacrylamide gels followed a specific immunofixation, they found 3 subtypes, Gc 1F-1F, 1F-1S and 1S-1S, in Gc 1-1 phenotypes and also 2 subtypes, Gc 2-1F and $2-1 \mathrm{~S}$, in Gc 2-1 phenotypes. Later, Constans et al. $(1978 \mathrm{a}, \mathrm{b})$ showed that this technique has a great resolving power to discriminate some $\mathrm{Gc}$ variants.

In the previous paper (Kuwata et al., 1978) we observed that a variant allele, $G c^{\mathrm{J}}$, attains to be a polymorphic frequency in Japanese populations. As mentioned in that paper, however, there were some cases in which it was somewhat difficult to distinguish the Gc J-1 from Gc 1-1 by agar gel immunoelectrophoresis used at that time. Therefore, we tried to re-examine this protein polymorphism by the immunofixation isoelectric focusing method. Omoto and Miyake (1978) already published their result on the distribution of Gc subtypes in this country. Our observation agrees with theirs as to the frequencies for the common alleles and also

\footnotetext{
1 This study was supported by the Scientific Research Grant (337023) from the Ministry of Education, Science and Culture of Japan.

Received April 26, 1979
} 
one of the variant alleles. Furthermore, it appears to show that there exists much complicated variation in Japanese populations, when larger samples are examined.

In this paper we report the result of re-analysis of the Gc J samples previously determined as well as a population survey in Japanese populations. Additional variants observed in the course of this study are also presented.

\section{MATERIALS AND METHODS}

The procedures for isoelectric focusing and immunofixation were essentially the same as those described by Constans et al. (1978a,b). The polyacrylamide gels of $1 \mathrm{~mm}$ thickness $(7.5 \% \mathrm{~T}, 3 \% \mathrm{C}, 2 \%$ Ampholine $\mathrm{pH} 4-6)$ were photopolymerized for $2 \mathrm{hr}$ in the presence of riboflavin. Serum samples were applied at the cathodal side of the gel using small rectangles of filter paper. Paper strips saturated with $1 \%$ phosphoric acid and $2 \%$ ethylenediamine were used for anode and cathode, respectively. Isoelectric focusing was performed with the LKB Multiphor or the apparatus manufactured by Joko Sangyo (Tokyo) under cooling. After $4 \mathrm{hr}$ separation period at $1,000 \mathrm{~V}$, the immunofixation was carried out using the monospecific anti-Gc (DAKO) with 1:3 dilutions. A strip of cellulose acetate membrane was soaked with the diluted antiserum and placed on the Gc region of the gel for a few minutes. Subsequently the strip was removed, washed overnight with saline and stained with Amido black 10B.

For further examinations some sera were also analysed by agar gel immunoelectrophoresis (Hirschfeld, 1962) and antigen-antibody crossed electrophoresis (Laurell, 1965) with the buffer system of polyacrylamide gels described by Kitchin (1965).

The serum samples with $\mathrm{Gc} J$ phenotypes previously determined by immunoelectrophoresis (Kuwata et al., 1978) were examined first in order to discriminate the variants from common $\mathrm{Gc}$ phenotypes. They were stored for up to 3 years after the initial use. For a population study sera newly collected from: (1) 103 medical students and 407 apparently healthy blood donors in Mie, western-central part of Japan; and (2) 229 medical students and 302 apparently healthy blood donors residing in and around Tokyo, were examined. Some family samples were also analysed, but only those with the variant subtypes are described in this paper.

\section{RESULTS}

\section{Heterogeneity of the Gc $J$}

In the previous study which was performed by prolonged immunoelectrophoresis (Kuwata et al., 1978), we identified the phenotypes with Gc J in 69 out of 1,347 individuals. Among them, 52 samples were re-examined by the immunofixation isoelectric focusing, and it was found that the $G c^{J}$ previously determined was subdivided into 4 different alleles. 

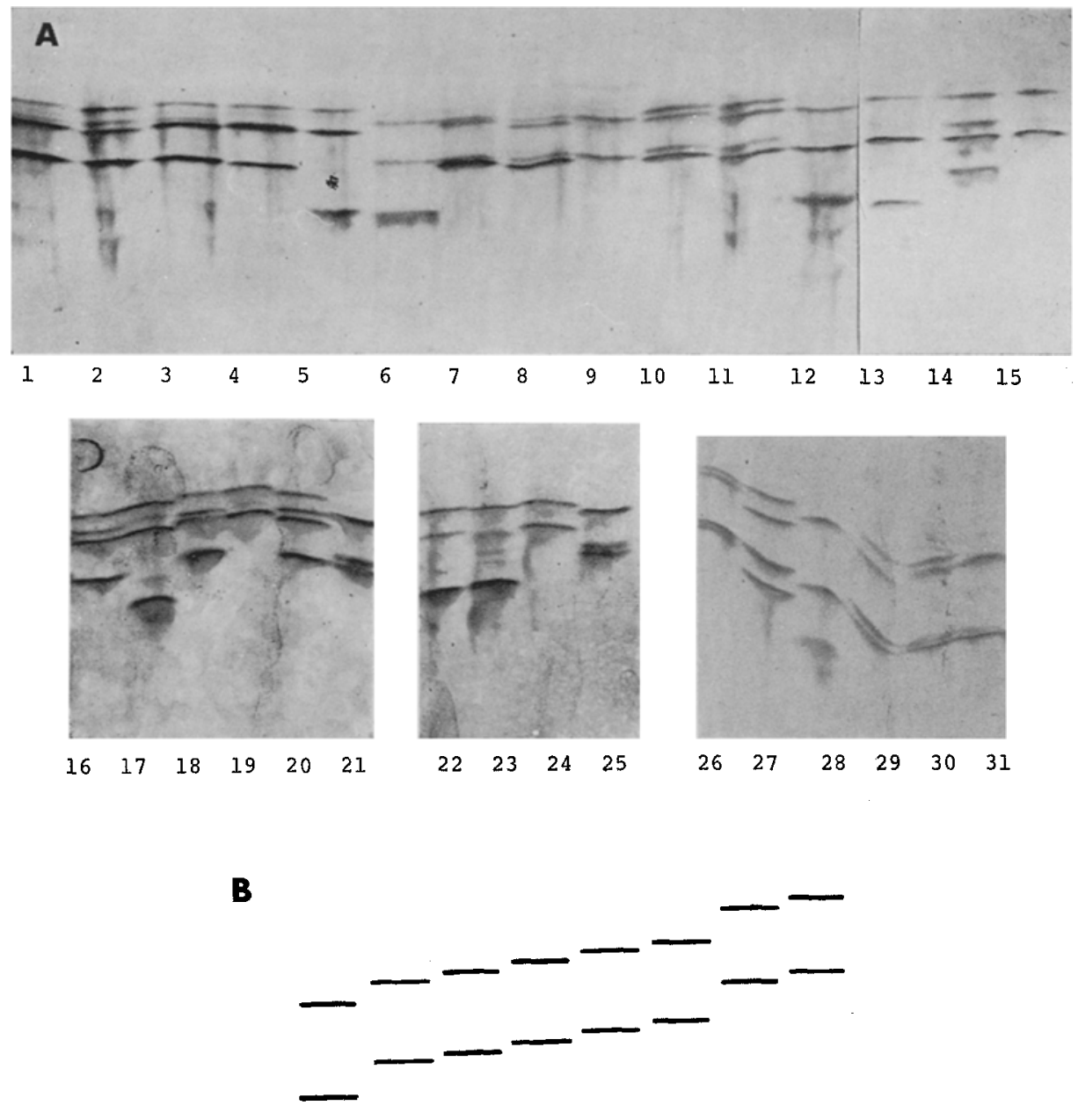

2 Mie ok 15 IF Ja Jb Jd Jc

Fig. 1. Immunofixation isoelectric focusing patterns in this study. (A) Photographs of cellulose acetate membranes. Samples $-1,3,18:$ Jc-1F, 2: Jd-1F, 4: Jc-1S, 5: Jc-2, 6, 23: Ja-2, 7: Ja-1F, 8: Ja-1S, 9: Ja-Ja, 10: Jb-1F, 11: Jb-1S, 12: 2-1S, 13: 2-1F, 14, 27: Mie-1F, 15: 1F-1F, 16, 20: Jd-1S, 17: Jd-2, 19: Jc-Jc, 21, 25, 26, 30:1F-1S, 22: Jb-2, 24: Jb-Jb, 28: Ok-2, 29: Ok-1F and 31: 1S-1S. (B) Scheme of the $\mathrm{Gc}$ bands produced by the 9 alleles observed in Japanese. 


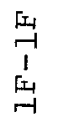
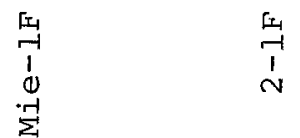

$\stackrel{N}{1}$

$n$
$\vdots$
$b$
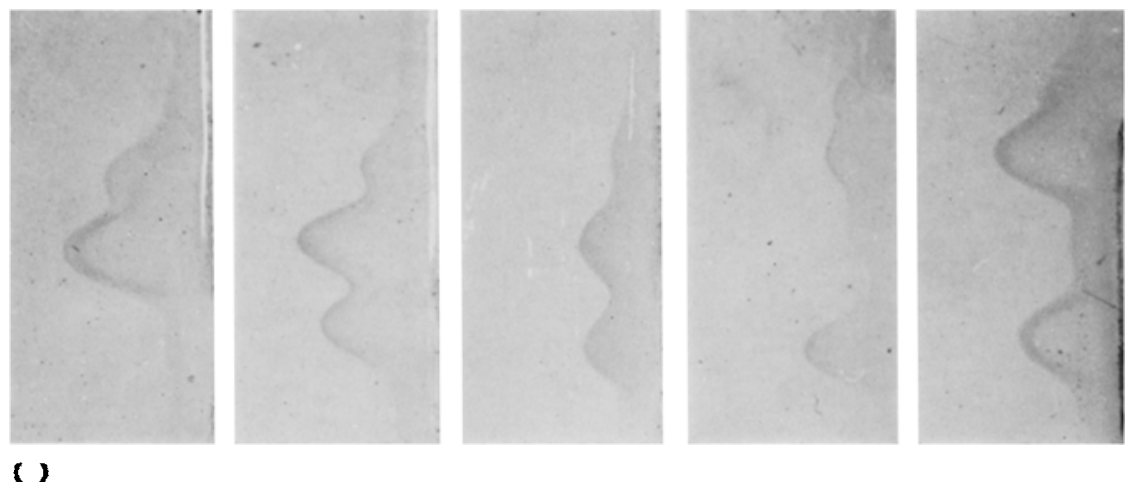

$\bar{\Xi}$

행응

$\ll \frac{8}{8}$

$\$ \ddot{m}$

官

范

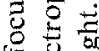

4 .

$5 \%$

可

$U$

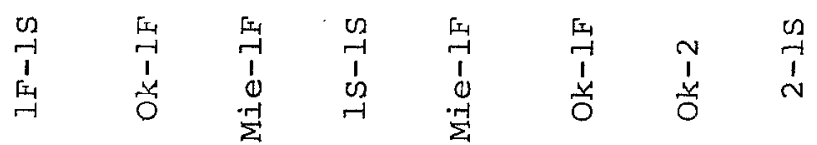

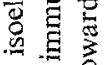

5

ㄴ.

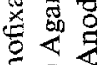

डิ

过的

正

宫

ष

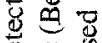

鸟

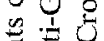

ज्ञ

후

8 过

要

宫

4 웡

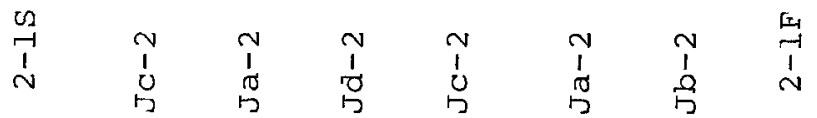

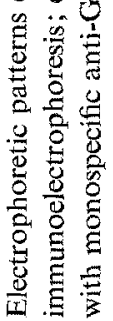

is

$\varangle$

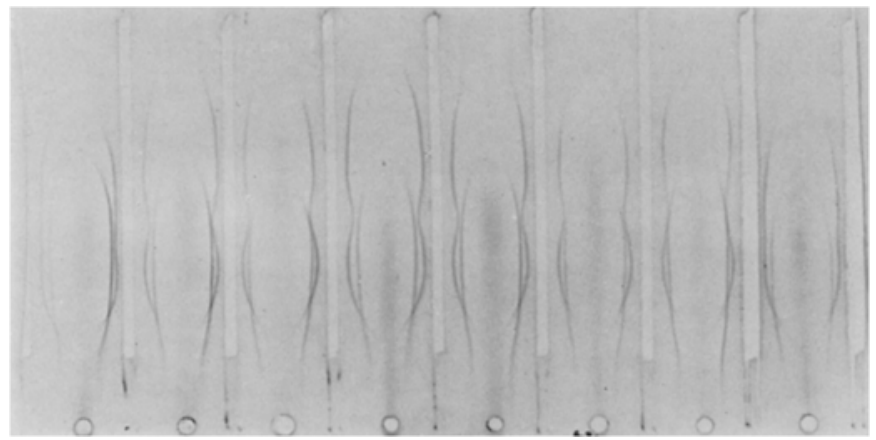


Table 1. Group-specific component (Gc) subtyping of the Gc J samples previously determined by immunoelectrophoresis.

\begin{tabular}{|c|c|c|}
\hline Previous type & No. & Subtype in this study \\
\hline $\mathrm{J}-\mathrm{J}$ & 3 & Ja-Ja 1, Jb-Jb 1, Jc-Jc 1 \\
\hline $\mathrm{J}-2$ & 16 & $\mathrm{Ja}-212, \quad \quad \mathrm{Jc}-24$ \\
\hline $\mathrm{J}-1$ & 33 & $\begin{array}{l}\text { Ja-1F 15, Jb-1F 2, Jc-1F 4, Jd-1F } 1 \\
\text { Ja-1S 7, Jb-1S 2, Jc-1S } 2\end{array}$ \\
\hline
\end{tabular}

Three samples of the Gc J-J showed double-band patterns, but their isoelectric points(IEP) were different from each other. In one case, the double bands were seen with slightly lower IEP than those of Gc $1 \mathrm{~F}$, and in another case the double bands were observed with lower IEP than those of the first one. The third case was found with both bands located much faster toward the anode. These three subtypes were provisionally named $\mathrm{Gc} \mathrm{Ja}-\mathrm{Ja}, \mathrm{Jb}-\mathrm{Jb}$ and $\mathrm{Jc}-\mathrm{Jc}$, respectively.

From 16 samples of the previous Gc J-2 phenotype two subtypes were detected, each having the double bands of $\mathrm{Gc} J a$ or $\mathrm{Jc}$ together with a $\mathrm{Gc} 2$ band.

Isoelectric focusing patterns from the previous Gc J-1 phenotype were somewhat complicated because of the presence of 4 bands in each case. However, a careful analysis by repeated and parallel runs enabled us to classify 7 subtypes in 33 samples, in which all but one had the double bands of $\mathrm{Gc} J a, J b$ or $\mathrm{Jc}_{\mathrm{c}}$ in addition to a set of $\mathrm{Gc} 1 \mathrm{~F}$ or $1 \mathrm{~S}$ proteins. In one case, the double bands of slightly higher IEP than those of Gc Jc were observed together with the double bands of Gc 1F. This type was tentatively called $\mathrm{Gc} \mathrm{Jd}-1 \mathrm{~F}$.

The immunofixation patterns obtained in this study are shown in Fig. 1, and the result of subtyping among the selected samples is given in Table 1. It is remarkable that the immunofixation isoelectric focusing permits the distinction of 12 different subtypes among three phenotypes distinguished by the ordinary immunoelectrophoresis. As mentioned later, they are genetically determined by 4 variant alleles, $G c^{\mathrm{Ja}}, G c^{\mathrm{Jb}}, G c^{\mathrm{Jc}}$ and $G c^{\mathrm{Jd}}$, in combination with one of the common alleles, $G c^{1 \mathrm{~F}}$, $G c^{1 \mathrm{~s}}$ and $G c^{2}$.

Some electrophoretic patterns by immunoelectrophoresis and crossed electrophoresis are shown in Fig. 2. As seen in the figure, the precipitation lines were almost indistinguishable between the samples of Gc Ja-2, Jb-2, Jc-2 and Jd-2. On the other hand, the profiles of crossed electrophoresis were quite different between Gc Ja-2 and Jc-2: both Gc Ja-2 and Jc-2 had 3 peaks, but the 2 peaks for $\mathrm{Gc}$ Ja were approximately equal and less pronounced than the Ge 2 peak, while in the case of Gc Jc-2, the middle peak showed much more pronounced than the faster one and also the Ge 2 .

\section{Other variants demonstrated by isoelectric focusing}

In the course of this study by the immunofixation isoelectric focusing 2 variants other than those described above were identified, which are also shown in Fig. 1. 
One variant had a 4-band pattern, 2 of them corresponding to $\mathrm{Gc} 1 \mathrm{~F}$ bands whereas the other 2 were located in a more cathodal position than the Gc 1S bands. This variant subtype was first detected in a student at Mie Univ. during a population study, and called Gc Mie-1F. Later, the same type was encountered in another student in Tokyo, who is not related to the former family. The family examinations revealed that the same variants were shared in other members of both families.

Table 2. Group-specific component (Gc) subtypes and gene frequencies in the Japanese.

\begin{tabular}{|c|c|c|c|c|c|}
\hline \multirow{2}{*}{ Sample group } & \multirow{2}{*}{$\frac{\text { Mie }}{\text { No. obs. }}$} & \multirow{2}{*}{$\begin{array}{c}\text { Tokyo } \\
\text { No. obs. }\end{array}$} & \multicolumn{3}{|c|}{ Combined } \\
\hline & & & No. obs. & $\%$ & No. exp. \\
\hline \multicolumn{6}{|l|}{ Gc subtype } \\
\hline $1 F-1 F$ & 112 & 120 & 232 & 22.29 & 235.31 \\
\hline $1 \mathrm{~F}-1 \mathrm{~S}$ & 120 & 128 & 248 & 23.82 & 237.93 \\
\hline $1 \mathrm{~S}-1 \mathrm{~S}$ & 29 & 27 & 56 & 5.38 & 60.15 \\
\hline $2-1 F$ & 117 & 131 & 248 & 23.82 & 251.78 \\
\hline $2-1 S$ & 60 & 68 & 128 & 12.30 & 127.29 \\
\hline $2-2$ & 40 & 28 & 68 & 6.53 & 67.35 \\
\hline Ja-1F & 6 & 14 & 20 & 1.92 & 19.48 \\
\hline Ja-1S & 4 & 3 & 7 & 0.67 & 9.85 \\
\hline $\mathrm{Ja}-2$ & 7 & 5 & 12 & 1.15 & 10.48 \\
\hline $\mathrm{Ja}-\mathrm{Ja}$ & 1 & 0 & 1 & 0.10 & 0.40 \\
\hline $\mathrm{Jb}-1 \mathrm{~F}$ & 1 & 0 & 1 & 1.10 & 1.88 \\
\hline $\mathrm{Jb}-1 \mathrm{~S}$ & 2 & 1 & 3 & 0.29 & 0.95 \\
\hline $\mathrm{Jc}-1 \mathrm{~F}$ & 5 & 2 & 7 & 0.67 & 6.23 \\
\hline$J_{C}-1 S$ & 1 & 1 & 2 & 0.19 & 3.15 \\
\hline $\mathrm{Jc}-2$ & 4 & 0 & 4 & 0.38 & 3.33 \\
\hline $\mathrm{Jd}-1 \mathrm{~F}$ & 0 & 1 & 1 & 0.10 & 0.99 \\
\hline $\mathrm{Jd}-2$ & 0 & 1 & 1 & 0.10 & 0.53 \\
\hline Others & 0 & 0 & 0 & 0 & 1.97 \\
\hline Variant* & 1 & 1 & 2 & 0.19 & - \\
\hline Total & 510 & 531 & 1,041 & 100.00 & $1,038.99$ \\
\hline \multirow[t]{3}{*}{$\mathrm{Chi}^{2}$} & 12.24 & 9.09 & & 10.676 & \\
\hline & $15 \mathrm{df}$ & $21 \mathrm{df}$ & & $21 \mathrm{df}$ & \\
\hline & $p>0.50$ & $\mathrm{p}>0.975$ & & $p>0.95$ & \\
\hline \multicolumn{6}{|l|}{ Gene } \\
\hline$G c^{1 F}$ & 0.465 & 0.487 & \multicolumn{3}{|c|}{$0.4759 \pm 0.0110$} \\
\hline$G c^{1 \mathrm{~S}}$ & 0.241 & 0.241 & \multicolumn{3}{|c|}{$0.2406 \pm 0.0094$} \\
\hline$G c^{2}$ & 0.263 & 0.246 & \multicolumn{3}{|c|}{$0.2546 \pm 0.0096$} \\
\hline$G c^{J a}$ & 0.019 & 0.021 & \multicolumn{3}{|c|}{$0.0197 \pm 0.0030$} \\
\hline$G c^{\mathrm{Jb}}$ & 0.003 & 0.001 & \multicolumn{3}{|c|}{$0.0019 \pm 0.0010$} \\
\hline$G c^{\mathrm{Jc}}$ & 0.010 & 0.003 & \multicolumn{3}{|c|}{$0.0063 \pm 0.0017$} \\
\hline$G c^{J d}$ & 0 & 0.002 & \multicolumn{3}{|c|}{$0.0010 \pm 0.0007$} \\
\hline
\end{tabular}

\footnotetext{
* Mie-1F, excluded for the calculation of gene frequencies.
} 
The immunoelectrophoretic pattern of Gc Mie-1F gave the precipitation line in the region intermediate between those of Gc 1-1 and 2-1, and the crossed electrophoresis showed 3 peaks in which the middle one was more pronounced than the others (Fig. 2).

The other variant was also a double-band variant. They had slightly higher IEP than those of Gc 1S. This variant was found in a family of a paternity case in Tokyo, and tentatively called Gc Okada (Ok) after the family name. The father and son had Gc Ok-1F and Ok-2, respectively (Fig. 1). Gc Ok was observed to be indistinguishable from Gc 1 on immunoelectrophoresis (Fig. 2).

\section{Distribution of Gc subtypes in Japanese population}

For a population study 1,041 sera from 2 local groups were examined. As indicated in Table 2, 18 subtypes corresponding to the presence of 8 alleles were identified. The distribution of $\mathrm{Gc}$ subtypes and $\mathrm{Gc}$ alleles in the 2 local groups is very similar and a good agreement between observed and expected numbers of the phenotypes under Hardy-Weinberg equilibrium is obtained for each local group as well as the total sample.

In the present series 6 common phenotypes were observed in about $94.1 \%$ of the samples while so-called rare variant phenotypes were detected in nearly $5.9 \%$. This proportion is compatible with the previous observation (rare phenotypes in $5.1 \%$ out of 1,347 individuals) by immunoelectrophoresis (Kuwata et al., 1978). Furthermore, 4 variant alleles determined from the selected samples in the previous study were

Table 3. Data on the families with variant Gc subtypes.

\begin{tabular}{|c|c|c|c|}
\hline Family & Propositus & Parent & Offspring \\
\hline Mat & Ja-Ja & Ja-1F $\times$ Ja-1S & Ja-Ja*, Ja-1F \\
\hline $77-84$ & $\mathrm{Ja}-\mathrm{Ja}$ & Ja-2 $\times$ Ja-1S & $\mathrm{Ja}_{\mathrm{J}} \mathrm{J}^{*}, 2-1 \mathrm{~S}, 2-1 \mathrm{~S}$ \\
\hline Mae & $\mathrm{Ja}-2$ & $2-1 F \times J a-1 F$ & Ja-2* \\
\hline Fur & $\mathrm{Ja}-2$ & Ja-1S $\times 2-1 S$ & $\mathrm{Ja}-2^{*}$ \\
\hline FuAs & Ja-2 & $\mathrm{Ja}-2 \times 2-2$ & Ja-2* \\
\hline Taka & $\mathrm{Ja}-2$ & Ja- $2^{*} \times 1 \mathrm{~F}-1 \mathrm{~F}$ & $2-1 F$, Ja-AF \\
\hline $\mathrm{T} 7752$ & $\mathrm{Ja}-1 \mathrm{~F}$ & $\mathrm{Ja}-2 \times 1 \mathrm{~F}-1 \mathrm{~F}$ & $2-1 F$, Ja-1F*, Ja-1F \\
\hline Iso & $J a-1 F$ & Ja-1F* $\times 2-1 S$ & 1F-1S, IF-1S, Ja-2 \\
\hline T7774 & Ja-1S & $\mathrm{Ja}-2 \times 1 \mathrm{~F}-1 \mathrm{~S}$ & Ja-1S*, Ja-1S \\
\hline $\mathrm{Om}$ & $\mathrm{Jb}-\mathrm{Jb}$ & $\mathbf{J b}-\mathrm{Jb}^{*} \times 2-1 \mathrm{~F}$ & $\mathrm{Jb}-2, \mathrm{Jb}-2, \mathrm{Jb}-2$ \\
\hline $77-48$ & $\mathrm{Jb}-1 \mathrm{~F}$ & $1 F-1 F \times J b-1 F$ & $\mathrm{Jb}-1 \mathrm{~F}^{*}$ \\
\hline $77-75$ & $\mathrm{Jc}-2$ & $\mathrm{Jc}-2 \times 2-2$ & $\mathrm{Jc}-2^{*}$ \\
\hline $78-13$ & $\mathbf{J}_{\mathrm{c}-1 \mathrm{~F}}$ & $1 \mathrm{~F}-1 \mathrm{~F} \times \mathrm{Jc}-1 \mathrm{~F}$ & $J c-1 F^{*}, 1 F-1 F$ \\
\hline Suzu & $\mathrm{J}_{\mathrm{C}-1 \mathrm{~F}}$ & $2-1 S \times J_{C}-1 F^{*}$ & $\mathrm{Jc}-2,2-1 \mathrm{~F}$ \\
\hline $\mathrm{T} 7736$ & Jd-1F & Jd-1S $\times 1 \mathrm{~F}-1 \mathrm{~S}$ & Jd-1F, Jd-1F* \\
\hline 77.55 & Mie-1F & $\dagger \times 1 \mathrm{~F}-1 \mathrm{~F}$ & Mie-1F, Mie-1F* \\
\hline $\mathrm{T} 7556$ & Mie-1F & Mie-1F $\times 2-1 F$ & Mie-1F*, Mie-1F \\
\hline Okada & Ok-2 & Ok-1F $\times 2-1 F$ & $\mathrm{Ok}-2^{*}$ \\
\hline
\end{tabular}

\footnotetext{
* Indicates the propositus.
} 
again observed in the new series, indicating that such variant alleles are widely distributed in Japanese populations.

As to the distribution of Gc subtypes in Japan, Omoto and Miyake (1978) already published their result in 310 individuals in Tokyo. The allele frequencies given in Table 2 are very similar to their figures for common alleles as well as $G c^{\text {Ja }}$ $\left(G c^{1 \mathrm{~F}} 0.466, G c^{1 \mathrm{~S}} 0.259, G c^{2} 0.257\right.$ and $\left.G c^{\mathrm{J}} 0.018\right)$. In addition, they observed 4 cases of the Gc 1F-X, which are probably identical to the present Gc Jc-1F.

\section{Family studies}

The family data of the Ge variants are summarized in Table 3. Although the numbers analysed are still not large, it is apparent that the 6 variant alleles determined in this study are all transmitted from parents to offspring in a simple Mendelian manner.

\section{DISCUSSION}

This study further confirms that the immunofixation isoelectric focusing possesses a resolving power much superior to the ordinary immunoelectrophoresis once employed frequently to examine Gc polymorphism. Indeed, this method revealed an unexpected degree of $\mathrm{Gc}$ variation in Japanese population, and we could identify a total of 24 phenotypes determined by 9 alleles in more than 1,000 sera. The present study was conducted independently in 2 laboratories, some samples of variants being exchanged, and got to identical results, although the differences to be disclosed are rather subtle on the immunofixation pattern.

The nomenclature for $\mathrm{Gc}$ variants seems to be confusing, since names of the locality where a mutant was first discovered or of the ethnic group characteristic for a variant have been widely accepted before, while numbers with a subscript like la or $2 \mathrm{~b}$ are also used in recent literatures. Therefore, the notations given in the above section are tentative and only valid for explanation in this paper.

Among the 6 variants described, judging from the electrophoretic patterns and frequencies in the population, we have no doubt that the present $\mathrm{Gc} \mathrm{Ja}$ is identical with the original Gc $J$ reported by Omoto et al. (1972). Furthermore, the Gc $J c$ is very likely to be the same as Gc Am (Amerindian) which was observed in South American Indians from Bolivia (Constans et al., 1978a), judging from their peculiar patterns in immunofixation isoelectric focusing and in crossed electrophoresis. Recently, Cleve et al. (1978) reported the presence of $3 \mathrm{Gc}$ variants in European populations. From the figures in their paper, the present Gc Jd and Gc Ok seem to correspond to their $\mathrm{Gc} \mathrm{V}^{2}$ and $\mathrm{V}^{1}$, respectively, both observed from Germans in Munch.

In the field of forensic medicine, the Gc system has been utilized as a genetic marker for paternity tests. The exclusion chance of a falsely alleged father was thus far calculated to be $16.2 \%$ by the three-allele model $\left(G c^{1}, G c^{2}\right.$ and $\left.G c^{\mathrm{J}}\right)$. Now, when the subtyping is carried out, the exclusion rate goes over $38.2 \%$ based on the 4 -allele 
model $\left(G c^{1 \mathrm{~F}}, G c^{1 \mathrm{~s}}, G c^{2}\right.$ and $\left.G c^{\mathrm{var}}\right)$ in Japanese. It is clear that the Gc subtyping is a valuable aid in the examination of cases of disputed paternity.

Acknowledgment We are indebted to Miss Eiko Nakazawa for her excellent technical assistance.

\section{REFERENCES}

Cleve, H., Patutschnick, W., Nevo, S., and Wendt, G. G. 1978. Genetic studies on the Gc subtypes. Human Genet. 44: 117-122.

Constans, J. and Viau, M. 1977. Group-specific component: Evidence for two subtypes of the $\mathrm{Gc}^{1}$ allele. Science 198: 1070-1071.

Constans, J., Viau, M., Cleve, H., Jaeger, G., Quilici, J. C., and Palisson, M. J. 1978a. Analysis of the $\mathrm{Gc}$ polymorphism in human populations by isoelectrofocusing on polyacrylamide gels. Demonstration of subtypes of the $\mathrm{Gc}^{1}$ allele and of additional $\mathrm{Gc}$ variants. Human Genet. 41: 53-60.

Constans, J., Viau, M., Pison, G., and Langaney, A. 1978b. Gc subtypes demonstrated by isoelectric focusing: further data and description of new variants among an African sample (Fula) from Senegal. Jap. J. Human Genet. 23: 111-117.

Hirschfeld, J. 1962. The Gc-system. Prog. Aller. 6: 155-186.

Kitchin, F. D. 1965. Demonstration of the inherited serum group-specific protein by acrylamide electrophoresis. Proc. Soc. Exp. Biol. Med. 118: 304-307.

Kuwata, M., Ishimoto, G., and Nakajima, H. 1978. Group-specific component (Gc) polymorphism in Japanese: an investigation on the phenotypic distribution with regard to the $\mathrm{GcJ}_{\mathrm{J}}$ allele. Jap. J. Human Genet. 23: 127-132.

Laurell, C. B. 1965 . Antigen-antibody crossed electrophoresis. Anal. Biochem. 10: 358-361.

Omoto, K., Cleve, H., and Misawa, S. 1972. A new variant phenotype of the group-specific component (Gc) found in a Japanese family. Jap. J. Human Genet. 17: 1-9.

Omoto, K. and Miyake, K. 1978. The distribution of the group-specific component (Gc) subtypes in Japanese. Jap. J. Human Genet. 23: 119-126. 